Signal \& Image Processing : An International Journal (SIPIJ) Vol.3, No.1, February 2012

\title{
Investigations of the MRI Images using Aura Transformation
}

\author{
Parveen Lehana ${ }^{1}$, Swapna Devi ${ }^{2}$, Satnam Singh $^{3}$, Pawanesh Abrol $^{4}$, Saleem \\ $\mathrm{Khan}^{1}$, Sandeep Arya ${ }^{1^{*}}$ \\ ${ }^{1}$ Dept. of Physics \& Electronics, University of Jammu, Jammu \\ ${ }^{2}$ Dept. of Electronics \& Communication, NITTTR Chandigarh \\ ${ }^{3}$ Dept. of Electronics \& Communication, SSCET, Badhani, Pathankot \\ ${ }^{4}$ Dept. of Computer Science, University of Jammu, Jammu \\ snp09arya@gmail.com
}

\begin{abstract}
The quality of biomedical images can be enhanced by using several transformations reported in the literature. The enhanced images may be useful to extract the exact information from these scans. In recent times, researchers exploited various mathematical models to smoothen and enhance the quality of the biomedical images with an objective to extract maximum useful medical information related to functioning or malfunctioning of the brain. Both real and non-real time based techniques have been explored and reported for this purpose. In this proposed work, aura based technique has been investigated for enhancing the quality of magnetic resonance imaging (MRI) scans of the human brain. The aura transformation based algorithm with some modifications has been developed and the performance of the algorithm is evaluated on a series of defected, diseased, and normal MRI brain images.
\end{abstract}

Keywords : MRI, image segmentation, brain tumor, aura transition, image processing.

\section{INTRODUCTION}

Image processing is a significant technique in several areas such as video coding, computer vision, and medical imaging [1] [2]. In medical imaging, for the successful treatment of lesions, tumors and vascular malformations in brain, MRI scans are vital. For further investigations, the deformable model images and projection of structures from brain onto MRI image is a commonly used [3]. These scans help in the diagnosis of injury for improving the results of surgical, radiosurgical, or radio-therapeutic treatment [4]. Converting an image into homogeneous regions is an area of research from a decade, especially when the image is made up of complex textures. A number of techniques have been proposed for this task, including spatial frequency techniques [5] [6]. Image processing techniques have been used widely depending on the specific application and image modalities. Computer based detection of abnormal growth of tissues in a human brain are preferred to manual processing methods in the medical investigations because of accuracy and satisfactory results. In MRI scans, the important task is to label the voxels according to their tissue type which includes white matter (WM), gray matter (GM), cerebrospinal fluid (CSF), and sometimes pathological tissues like tumor [7] [8]. Several methods for processing the MRI scans

DOI : 10.5121/sipij.2012.3109 
Signal \& Image Processing : An International Journal (SIPIJ) Vol.3, No.1, February 2012

have been developed. The different methods of analyzing the scans can be classified under five broad categories. These are methods based on statistics (clustering methods) [9], fuzzy sets theory [10], mathematical morphology [11], edge detection [12], and region growing [13]. Image processing of a brain image allows extracting the lesions from the brain data and provides information for further stages of the quantitative assessment. A lot of techniques have been proposed for processing MRI scans for the purpose of effective diagnosis [14-16]. However, there is still scope for improvement in terms of quality of the processed images. As a lot of information is embedded in the texture of the MRI scans, the analysis of the texture of MRI scan may be quite significant for predicting the brain related problems.

Recently, a technique called aura introduced by Rosalind W. Picard and Ibrahim Elfadel [17-19] has been reported and applied for the analysis of the textures, restoration of the distorted images and segmentation of geometrical patterns [20-25]. Even though many techniques have been used for analysis of MRI images, the extensive use of aura based techniques for enhancing the content of the scans has not been carried out.

In this research work, an aura based technique is investigated for enhancing the quality of an MRI scans for better medical diagnosis. Extensive investigations have been carried out with the MRI scans of several subjects involving different types of brain related problems. The analysis of the processed images indicates the enhancement of the important regions of the brain in the MRI scan responsible for the abnormalities in the functioning of the brain. For employing the standard model reported by Rosalind W. Picard and Ibrahim Elfadel [17-19], to cover gray scale images efficiently, few modifications have also been incorporated. The details of MRI have been presented in the next section. In the subsequent sections, Aura transformation, mathematical background of aura, methodology, and results are discussed.

\section{MAGNETIC RESONANCE IMAGING (MRI)}

Magnetic resonance imaging (MRI) is a significant technique of examining the human body. MRI helps to explain and characterize the neural architecture of the human brain. It is a non-invasive method of obtaining images corresponding to the sections of the specific structure in the human body. MRI scanner employs a magnetic field and radio waves to generate exhaustive images of the human brain [26]. MRI data is most relevant in the studies of a head, specifically, for tracking the size of brain tumor and other brain related problems. MRI helps for early detection of intracranial tumors and precise estimation of tumor boundaries, which are significant when planning radiotherapy. Analytical imaging of MRI scan has also been used to assess the maturity of the central nervous system and diagnose malformations. The resonance is also indispensable for imaging of vascular changes. Using this method of diagnostic, imaging allows obtaining information about aneurysms and accompanying symptoms. MRI also helps in showing seditious changes of the central nervous system and gives accurate assessment of the degree of brain atrophy [27]. The automatic classification of brain MRI may be used for identifying regions having various brain diseases like cerebro-vascular, alzheimer, brain tumor, inflammatory, etc. Although automatic classification is a challenging process and a lot of research is being carried out in this direction involving different techniques. These techniques may be broadly classified in two categories supervised and unsupervised. Some of the techniques in supervised classification are artificial neural networks, support vector machine (SVM), and $k$ nearest neighbor $(k-\mathrm{NN})$. In unsupervised classification self-organization map (SOM) and fuzzy $c$-means are included [2830]. The MRI images provide necessary information about the soft tissue anatomy that is very 
Signal \& Image Processing : An International Journal (SIPIJ) Vol.3, No.1, February 2012

useful for the diagnosis and treatment in brain pathology. MRI techniques are still in the development stage, and most of the research is concentrated around the improvement of the quality and speed of acquisition of MRI signal. Several processes involved in the interpretation of MRI signals are manual. Therefore, even in the laboratories having the state of art technologies there is a necessity for automated image processing technique for MRI based medical diagnosis.

\section{AURA TRANSFORMATION}

The concept of aura set, aura measure and aura matrix was introduced by Rosalind W. Picard and Ibrahim Elfadel for analysis and synthesis of textures [17] [18]. Aura is defined as the relative distribution of pixels intensities with respect to a predefined structuring element. The matrix computed from the local distribution of pixel intensities of the given texture is called aura matrix. Aura set and aura measure are the basic components of the aura based texture analysis. Aura Set describes the relative presence of one gray level in the neighborhood of another gray level in a texture and its quantitative measure called aura measure. Xuejie Qin and Yee Hong Yang also used the aura based framework and showed that basic gray level aura matrices (BGLAM) can uniquely represent the given texture [19]. A neighborhood element is used to calculate the relative presence of one gray level with respect to another. The concept of Aura has also been applied to 3D textures to generate the solid textures from the input samples automatically without user intervention. The results computed using weighted-aura matrix distance, outperform Wei and Levoy's method and are comparable to that proposed by Jagnow, et. al [31].

While considering several approaches, we studied previous methodologies that have been developed and improved upon by various authors to computationally analyze images [19] [21-23] [32-34]. Present work is based on recently proposed BGLAM (Basic Gray-Level Aura Matrices) mathematical framework [19], which is developed based on the aura concepts (i.e., aura sets, aura measures, and aura matrices) [35]. The aura based transformation for texture analysis and synthesis may have many advantages. The main advantage is that the input texture image is not needed once the aura matrix has been calculated. This reduces the storage and computational complexity. The other advantage is that aura matrix of any texture is independent of its orientation. Hence each texture can be uniquely represented. In addition, this technique may further be used for constructing aura based distance measures for comparing the textures. The concept of aura can also be explored for implementing different types of digital filters to estimate the boundary of different components in images of solids and fluids [27].

The output texture can be generated by the concatenation of the input texture sample that introduces a very serious problem of propagating the distortions present in the input sample to the synthesized texture. The sources of distortion may be due to the presence of random noise, wrinkles, foldings, cracks, rusting etc. Further, the problem is inflated if these distortions are present near the edges. The net result of these distortions may be the unpleasing and distracting effects developed in the synthesized output texture [27].

\section{MATHEMATICAL BACKGROUND OF AURA}

As discussed earlier, aura is a significant technique for analysis and synthesis of textures. The mathematical framework of aura is defined in [17-19] [21-24]. The input texture is represented as an ordered set of pixel intensity values. It is denoted by $\mathrm{S}$ and has the size $\mathrm{N} \mathrm{x} \mathrm{M}$. The structuring element is a collection of pixel intensities taken from site where it is placed on the input texture 
Signal \& Image Processing : An International Journal (SIPIJ) Vol.3, No.1, February 2012

image. After placing this element at a particular site, the intensity values taken from two sets called Set A and Set B are matched with the pattern of the pixel values of the image just below the structuring element. The choice of the shape of the structuring element depends upon the nature of analysis and the input given image. The values of the intensities used for A and B depend upon the input texture image and the nature of the analysis. Various schemes for allotting the intensity values to set A and B lead to different aura patterns. In general, the structuring element is denoted by $\mathrm{N}=\left\{\mathrm{N}_{\mathrm{s}}, \mathrm{s} \in \mathrm{S}\right\}$, where, $\mathrm{N}_{\mathrm{s}}$ is one of the elements (single pixel intensity) taken from the set $\mathrm{S}$ or its subset A or subset B. The elements around which the aura is to be determined are taken from the set A. The intensity values of the surrounding elements are taken from the set $\mathrm{B}$. The set of matching patterns in the given texture image as per the structuring element is called aura matrix. In another words, the aura of A w.r.t. B is given by

$$
\vartheta_{B}(A)=\bigcup_{s \in A}\left(N_{S} \cap B\right)
$$

The total number of aura patterns obtained for a combination of pixel intensity values taken from set $\mathrm{A}$ and set $\mathrm{B}$ according to the structuring element is called the aura measure for that combination and is given by

$$
m\left(P_{A}, P_{B}\right)=\sum_{P_{t}=P_{A B}}\left|P_{t} \cap P_{A B}\right|
$$

where $\left|P_{t} \cap P_{A B}\right|$ represents the total number of elements in the given set.

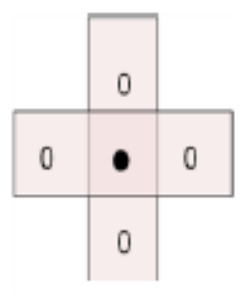

(a)

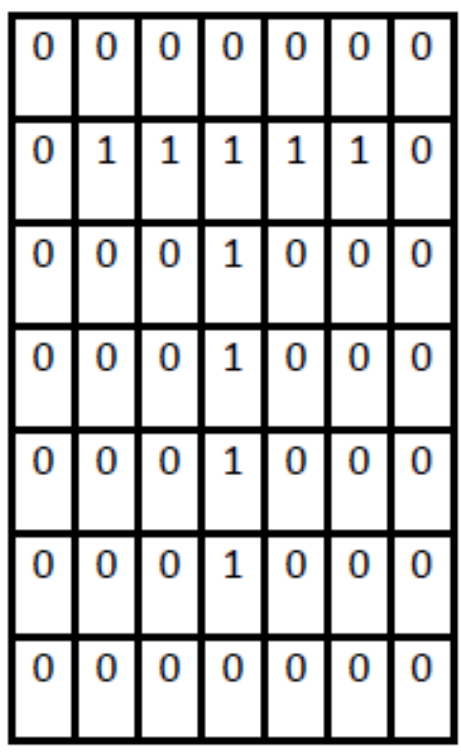

(b)

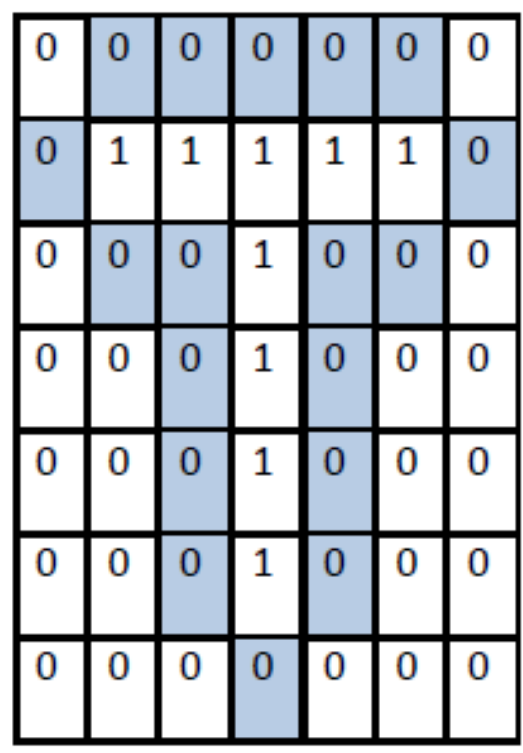

(c)

Fig. 1. Example of an aura on a binary lattice with four nearest neighbors system. (a) A sample binary lattice S, where the subset A is the set of all 1's and B the set of all 0's. (b)The structuring element of the neighborhood system (c) The set of shaded sites is the aura set of A with respect to B. 
Signal \& Image Processing : An International Journal (SIPIJ) Vol.3, No.1, February 2012

$\mathrm{P}_{\mathrm{A}}$ and $\mathrm{P}_{\mathrm{B}}$ are the patterns formed by taking elements from set $\mathrm{A}$ and set $\mathrm{B}$, respectively according to the structuring element. $\mathrm{P}_{\mathrm{t}}$ is the pattern formed in the input texture at the position where the structuring element is currently placed. $\mathrm{P}_{\mathrm{AB}}$ is the pattern formed from the elements set $\mathrm{A}$ and set $\mathrm{B}$. Hence the aura measure for a combination of $\mathrm{P}_{\mathrm{A}}$ and $\mathrm{P}_{\mathrm{B}}$ at a particular site in the input texture is equal to total number of matchings of all possible patterns formed from set A \& set B with respect to the pattern formed below the current position of the structuring element. It should be noted that the matching of patterns is to be computed only at the sites where the intensity values in the input texture are identical to the elements in set A.

The matrix of all the patterns which can be formed for different combinations of the intensity values taken from set $\mathrm{A}$ and $\mathrm{B}$ with respect to the given structuring element is called aura matrix. For simplicity the image textures are analyzed using their gray level representations. GLAM (Gray-Level Aura Matrix) defines the aura measure for a typical the structuring element and the texture image shown in Fig. 1 between $S_{i}$ and $S_{j}$ using the relation

$$
\left\lfloor a_{i, j}\right\rfloor=m\left\lfloor S_{i}, S_{j}\right\rfloor
$$

Where $\mathrm{Si}$ and $\mathrm{Sj}$ are the ith and $\mathrm{jth}$ gray levels in the range $\{0 \leq \mathrm{i}, \mathrm{j} \leq 1\}$

The standard mathematical formulation of aura described in this section is limited in application because of the need of exact matching of the gray values of structuring elements in with the image under processing. We have slightly relaxed some of the constraints of the standard aura and the modified algorithm is explained in the next section.

\section{METHODOLOGY}

It is clear from the literature survey that limited work has been carried out using the standard concepts of aura based transformation. Our previous investigations have shown that the power of aura transformation may be enhanced by modifying some of the concepts of aura technique [2125]. In the present research work, the basic concepts of aura are further modified to widen its application area. Here, modified aura concept has been used for investigating and enhancing the quality of MRI scans for better medical diagnosis. The modified aura based algorithm is shown in Fig. 2 in the form of a flow chart.

In preprocessing, the input MRI scan is converted to gray scale and its size is modified to reduce the number of computations. The reduction depends upon the expected size and texture of the abnormal region in the scan.

Different types of abnormalities are introduced in the preprocessed scans for investigating the effect of aura on these abnormalities and the neighborhood structures of the brain.The abnormalities like Alzheimer, calcium migraine and other growth of tissues called tumor were manually introduced. A neighborhood element is defined in the form of a $3 \times 3$ matrix.

The values of the elements of this matrix are estimated on the basis of gray scale values of the given MRI scan. The input image is processed using this structuring element by traversing it pixel by pixel on the whole image. At every placement, the differences of the gray scale values of the neighborhood elements and the corresponding pixels below it are computed. Depending upon the difference threshold $T d$, the $3 \times 3$ matrix of the differences is converted to zeros and ones. If the difference is less than $T d$, the corresponding element is mar ked as one otherwise, zero in the 
Signal \& Image Processing : An International Journal (SIPIJ) Vol.3, No.1, February 2012

difference matrix. If the total number of ones in the difference matrix is more than a threshold called matching threshold $\mathrm{Tm}$, the pixel corresponding to the central element of the neighborhood element is marked as black, otherwise left unchanged. This process is repeated for the entire input image. The investigations have been carried out with different values of both the thresholds and input MRI scan involving various abnormalities. The evaluation of the enhancement in the processed MRI scan with respect to the input image was carried out using the visual inspection.

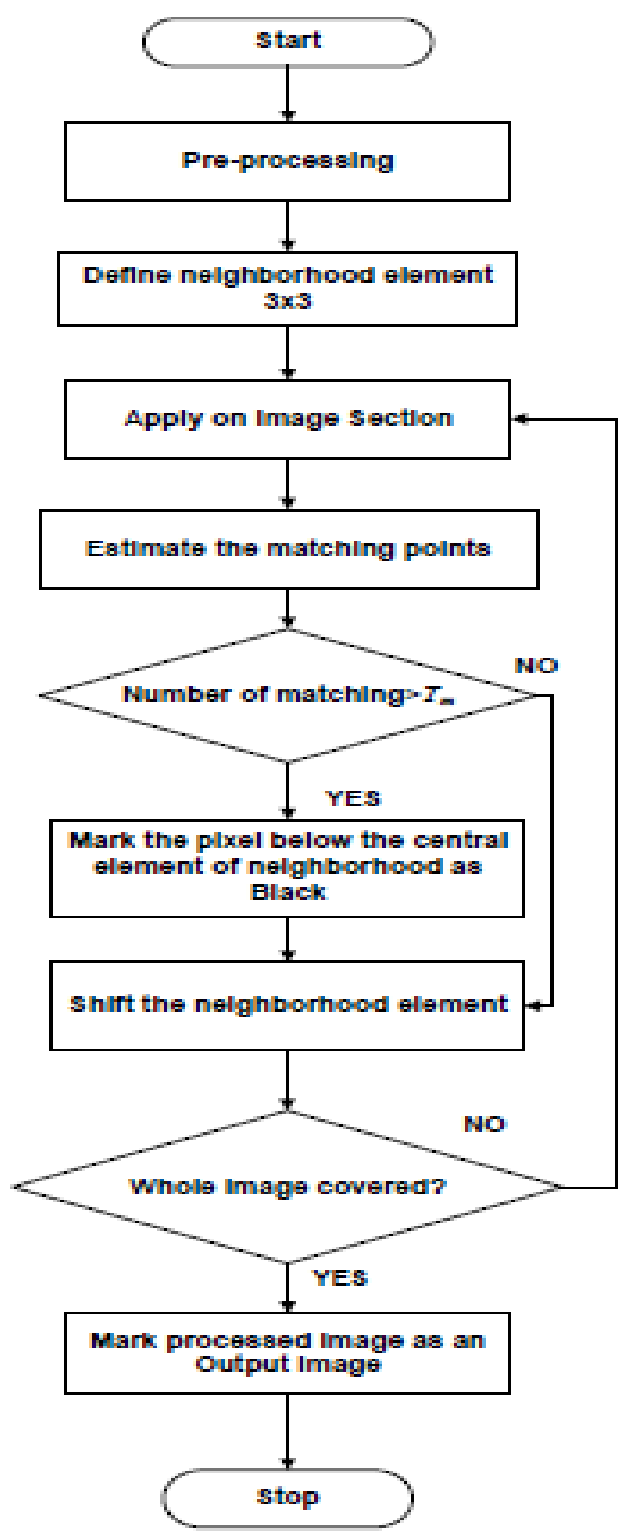

\section{RESULTS}

The investigations were carried out using two sets of MRI scans. The first set included the images where the abnormality was introduced manually with varying sizes, shapes, positions and textures. The second set included the scans having actual abnormalities corresponding to different subjects and diseases. 
Signal \& Image Processing : An International Journal (SIPIJ) Vol.3, No.1, February 2012

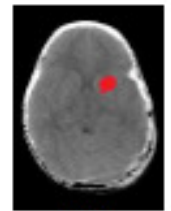

1(a)

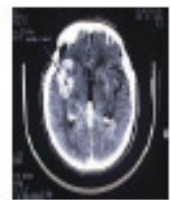

2(a)

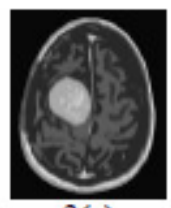

3(a)

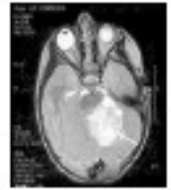

4(a)

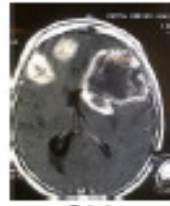

5(a)

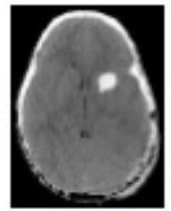

$1(\mathrm{~b})$

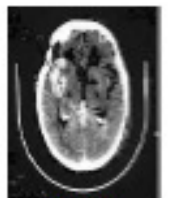

2(b)

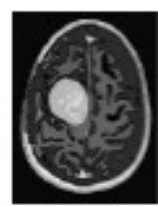

3(b)

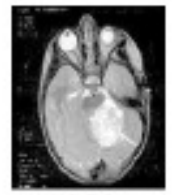

4(b)

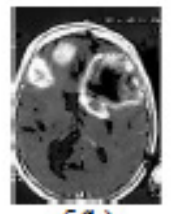

$5(b)$

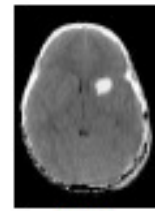

1(c)

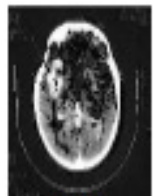

2(c)

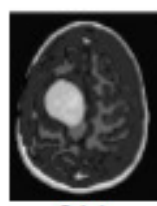

3(c)

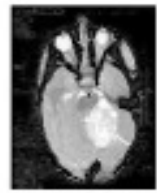

$4(c)$

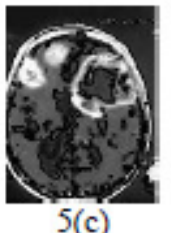

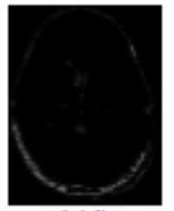

$1(\mathrm{~d})$

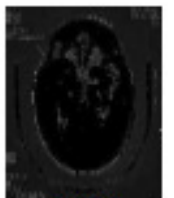

2(d)

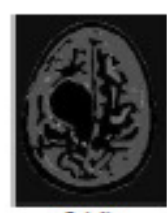

$3(d)$

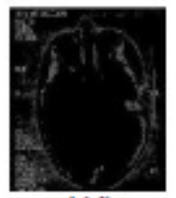

4(d)

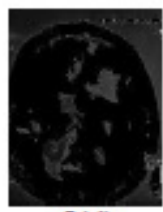

$5(d)$

Fig. 3. Input and the corresponding processed MRI scans with different types of abnormalities and threshold values.

The images in these two sets were processed as per the modified aura methodology described in the previous section. The results were obtained by modifying the values of the thresholds $T_{d}$ and $T_{m}$. Some of the processed images are shown in Fig. 3. The input images are shown in the column I. The value of $T_{d}$ for these images was fixed at $30 \%$ of the maximum intensity value in the image under consideration. The corresponding processed images for values of $T_{m}$ as 3,7 , and 9 are shown in columns II-IV, respectively.

The visual analysis of the processed images with respect to the input images shows that the enhancement is better for the moderate values of the matching threshold i.e. around $T_{m}=7$. As the value of $T_{m}$ increased beyond 7 , the quality of the processed images started deteriorating. Similarly, it was also observed that when the value of difference threshold $T_{d}$ was fixed in the range $25-30$ percent of the maximum intensity, better enhancement was achieved. 
Signal \& Image Processing : An International Journal (SIPIJ) Vol.3, No.1, February 2012

\section{CONCLUSION}

In this study, investigations were carried out to enhance the quality of the MRI scans involving different types of abnormalities using modified aura based framework. It was observed that modified framework with some constrains relaxed, is relatively less computationally expensive, simple, and promising. The investigations further showed that the processed MRI scans were enhanced in quality. The enhanced images may be used for predicting the abnormalities in brain more effectively and accurately. The investigations involving the images obtained from other medical imaging techniques are in our future plan.

\section{REFERENCES}

[1] L.P.Clarke, R.P. Velthuizen, M.A. Camacho, J.J. Heine, M. Vaidyanathan, L.O. Hall, R.W. Thatcher, and M.L. Silbiger , "MRI segmentation: methods and applications," Magn. Resonance Imag., vol. 13, no. 3, pp. 343-368, 1995.

[2] M. Kunt, A.Ikonomopoulos, and M. Kocher, "Second-generation image-coding techniques," in Proc. IEEE, Apr. 1985, vol. 73, no. 4, pp. 549-574.

[3] M. B. Cuadra, C. Pollo, A. Bardera, O. Cuisenaire, J. G. Villemure, and J. P. Thiran, "Atlas-based segmentation of pathological MR brain images Using a model of lesion growth," IEEE Trans. Med. Imag., vol 23, no 10, pp. 1301-1314, 2004

[4] V. P. Gladis, P. Rathi and S. Palani, "Detection and characterization of brain tumor using segmentation based on hsom, wavelet packet feature spaces and ann," in Proc. IEEE 3rd Int. Conf. ICECT, 2011, Kanyakumari, vol. 6, pp. 274-277.

[5] Y. M. Zhu and R. Goutte, "Analysis and comparison of space/spatialfrequency and multiscale methods for texture segmentation,” Opt. Eng., vol. 34, no. 1, pp. 269-282, Jan. 1995.

[6] A. K. Jain and F. Farrokhnia, "Unsupervised texture segmentation using Gabor filters," Putt. Recogn., vol. 24, no. 12, pp. 1167-1186, Dec. 1991.

[7] M. M. Ahmed and D. B. Mahamad, "Segmentation of brain MR images for tumor extraction by combining k-means clustering and Perona-Malik anisotropic diffusion model," Int. J. of Imag. Processing, vol. 2, no. 1, pp. $27-34,2008$.

[8] G. Mirajkar and B. Barbadekar, "Automatic segmentation of brain tumors from MR Images using undecimated wavelet transform and Gabor wavelets," in Proc. IEEE Trans. ICECS, 2011, Athens, pp. 702-705.

[9] S. Chen and D. Zhang, "Robust image segmentation using FCM with spatialconstraints based on new kernel induced distance measure," IEEE Systems, Man, and Cybernetics Society, vol. 34, pp. 10971916, 2004

[10] S. Shen, W. Sandham, M. Granat, and A. Sterr, "MRI fuzzy segmentation of brain tissue using neighborhood attraction with neural-network optimization," IEEE Trans. on Inf. Tech. in Biomed., vol. 9, pp. 459-467, 2005.

[11] R. Stokking, K. L. Vincken, and M. A. Viergever, "Automatic morphology-based brain segmentation (MBRASE) from MRI-T1 data,” NeuroImage, vol. 12, pp. 726-738, 2000. 
Signal \& Image Processing : An International Journal (SIPIJ) Vol.3, No.1, February 2012

[12] J. R. J. Alaniz, V. M. Banuelos, O. Y. Suarez, "Data-driven brain MRI segmentation supported on edge confidence and a priori issue information," IEEE Trans. on Med. Imag., vol. 25, pp. 74-83, 2006

[13] N. Kriegeskorte and R. Goebel, "An efficient algorithm for topologically correct segmentation of the cortical sheet in anatomical MR volumes," NeuroImage, vol. 14, pp. 329- 346, 2001.

[14] M. R. Kaus and S. K. Warfield, A. Nabavi, P. M. Black, F. A. Jolesz, and R. Kikinis, "Automated segmentation of MR images of brain tumors," Radiology, vol. 218, pp. 586-591, 2001.

[15] M. C. Clark, L. O. Hall, D. B. Goldgof, and R. Velthuizen, F. Reed Murtagh, and M. S. Silbiger, "Automatic tumor segmentation using knowledge-based techniques," IEEE Trans. on Med. Imag., vol. 17, pp. 187-201, 1999.

[16] M. Prastawa, E. Bullitt, S. Ho, and G. Gerig, "A brain tumor segmentation framework based on outlier detection,” Medical Image Analysis, vol. 8, pp. 275-283, 2004.

[17] R. W. Picard and I. Elfadel, "Structure of aura and co-occurrence matrices for the Gibbs texture model", J. Mathematical Imag. and Vision, vol. 2, pp. 5-25, 1992.

[18] R.W. Picard and I. Elfadel, "Gibbs random fields, co-occurrences, and texture modeling", IEEE Trans. Pattern Recognition and Machine Intelligence, vol. 16, no. 1, 1994

[19] X. Qin, Y. H. Yang, "Basic gray level aura matrices: theory and its applications to texture synthesis," in Proc.10th International Conference on Computer Vision, 2005.

[20] X. Qin and Y. H. Yang, "Aura 3D textures," IEEE Trans. Vis. Comput. Graphics, vol. 13, no.2, 2007.

[21] P. Abrol, D. Padha, and P. K. Lehana, "Application of aura in Identification of textural objects", PCTE J. Computer Science, vol.3, issue no.2, Jul-Dec, 2008.

[22] P. Abrol, D. Padha, and P. K. Lehana, "Effect of distortion on the synthesized textures using aura based techniques", in Proc. National Conf. Video, Cyber Learning and Electronics, 2008, NITTTR, Chandigarh.

[23] P. Abrol and D. Padha, "Textural visualization: algorithm and analysis", PCTE J. Computer Sciences, vol.3, issue no.1, pp. 54-58, Jan-June, 2007.

[24] P. Abrol, D. Padha, P.K. Lehana, and I. Gandotra, "Transmission of synthesized textures over low bandwidth networks", in Proc. AICTE sponsored National Conf. next Generation Computing and Inf. Sys., 2007, MIET, Jammu.

[25] P. Abrol, D. Padha, and P. K. Lehana, "Development of a dsp based textural visualization system", in Proc. UGC sponsored National Seminar on Data Mining Techniques \& Applications, Dept. of Computer Applications, 2007, M. C., Kerala, pp. 23-25.

[26] D. Weishaupt, B. Marincek, and V. D. Koechli, How does MRI work?: an introduction to the physics and function of magnetic resonance imaging. Springer, 2nd edition, 2003.

[27] Konsylium NZOZ, http://www.tomograf.com.pl/zakresbadan. php (in Polish), retrieved in April 2011.

[28] S. Chaplot, L. M. Patnaik, and N. R. Jagannathan, "Classification of magnetic resonance brain images using wavelets as input to support vector machine and neural network," Biomed. Signal Processing, vol. 1, pp. 86-92, Jun.2006. 
Signal \& Image Processing : An International Journal (SIPIJ) Vol.3, No.1, February 2012

[29] E. A. El-Dahshan, T. Hosny, and A. M. Salem, "Hybrid intelligent techniques for MRI brain images classification,” Digital Signal Processing, vol. 20, pp. 433-441, 2010.

[30] M. Maitra and A. Chatterjee, "Hybrid multiresolution slantlet transform and fuzzy c-means clustering approach for normal-pathological brain MR image segregation,” Med. Eng. Phys., Aug. 2007.

[31] A. Vailaya, M. Figueiredo, A. K. Jain, and H. J. Zhang, "Image classification for content-based indexing,” IEEE Trans. Image Processing, vol. 10, no. 1, pp.117-130, 2001.

[32] S. Abbasi and F. Mokhtarian, "Affine-similar shape retrieval: application to multiview 3-D object recognition," IEEE Trans. Image Processing, vol. 10, no. 1, pp.131-139, 2001.

[33] Y. Deng, B. S. Manjunath, C. Kenney, M. S. Moore, and H. Shin, "An efficient color representation for image retrieval,” IEEE Trans. Image Processing, vol. 10, no. 1, pp.140-147, 2001.

[34] I. Epifanio and G. Ayala, "A random set view of texture classification," IEEE Trans. Image Processing, vol. 11, no. 8, pp.859-867, 2002.

[35] I.M. Elfadel and R.W. Picard, "Gibbs random fields, cooccurrences, and texture modeling," IEEE Trans. Pattern Analysis and Machine Intelligence, pp. 24-37, 1994. 